\title{
Reduction of Electromagnetic Interference Susceptibility in Small Signal Analog Circuits using Complementary Split-Ring Resonators
}

\author{
Daniel Pérez, Ignacio Gil, Member, IEEE, Javier Gago, Raúl Fernández-García, Member, IEEE, Josep \\ Balcells, Senior Member, IEEE, David González, Member, IEEE, Néstor Berbel and Juan Mon
}

\begin{abstract}
Low frequency analog and digital electronic circuits are susceptible to electromagnetic interference in the radiofrequency $(\mathrm{RF})$ range. This disturbance is produced when the coupled RF signal is rectified by the non-linear behavior of the semiconductors used in the small signal analog input stage of the electronic system. Circuits based on operational amplifiers are usually employed for such input stages. These circuits present an AM demodulation produced by nonlinearity of internal transistors. Such a phenomenon generates demodulated signals in the low-frequency range. In this paper, this effect is suppressed by combining the conventional printed circuit board layout with complementary split ring resonators (CSRRs). CSRRs are constitutive elements for the synthesis of metamaterials with negative effective permittivity which are mainly excited to the host line by means of electric coupling. Electromagnetic simulations and experimental results show an effective rejection of the undesired RF demodulation effect with no-extra cost in terms of the device area or manufacturing process.
\end{abstract}

Index Terms - complementary split-ring resonators, electromagnetic interference, operational amplifier.

\section{INTRODUCTION}

$\mathrm{E}_{\mathrm{f}}^{\mathrm{L}}$ LECTRONIC SYSTEMS are disturbed by high frequency radiofrequency (RF) electromagnetic interference (EMI) noise, in which the amplitudes change randomly in time. This out of frequency band electromagnetic compatibility (EMC) issue is produced when the RF signal is coupled to sensors and cabling of the system, conducting the EMI to signal-conditioning, and causes errors or malfunction due to the rectification by the non-linear behavior of the semiconductors used in the electronic systems. In fact, signal conditioners or transducers are typically based on operational amplifiers (OPAMPs) operating in the linear mode. The nonlinearity of amplifier transistors causes an AM demodulation of RF signals (Fig. 1) [1]. When this random AM modulated RF noise reaches the inputs of an OPAMP, three significant effects are produced at the output [2]: First, a

Manuscript received April 4, 2011. This work was supported by the SpainMICINN under Project TEC2010-18550 and AGAUR 2009 SGR 1425.

The authors are with the Department of Electronic Engineering, Universitat Politècnica de Catalunya, Terrassa (Barcelona) 08222, Spain (e-mail: ignasi.gil@upc.edu). demodulated low frequency signal, at the modulating frequency, $f_{m}$, whose amplitude is proportional to the amplitude of the random modulating noise. These lower frequency components are derived from the amplitude envelope of the original RF signal. Second, a DC signal due to the rectification effect (caused by non-linearity). This effect is provoked by the nonzero average value of signal after the nonlinearity. Finally, several RF components due to the original RF carrier signal, centered at carrier frequency, $f_{c}$, and its harmonics, containing also lateral bands at $f_{c} \pm f_{m}$, due to AM demodulation. These three different components are illustrated in Fig. 1. The components of the last group, usually present certain attenuation, since they are located at the rejection band of amplifiers. However, DC and low-frequency components fall into the pass band of OPAMPs and contribute to a worsening of the signal/noise ratio, i.e., the interference signal cannot be distinguished from useful signal.

To solve this problem, effective filtering can be implemented at the input stages of the system (i.e., input connectors) in order to avoid the non-desired rectification. In order to overcome the limitations of standard EMI filters in terms of signal delay, printed circuit board (PCB) area, and cost, new filtering techniques are required. Several solutions based on increasing design robustness [3] or layout shielding improvements [4-5] have been proposed. Solutions based on design robustness, can typically involve higher number of electronic components, whereas layout shielding can involve extra metal layers or area. Recently, specific multilayer layout techniques based on electromagnetic band gaps (EBGs) have appeared, in order to filter EMI in several applications with good performance [6-10]. EBGs belong to a broad family of artificial media with electromagnetic properties generally not found in nature, called metamaterials. EBGs correspond to metamaterials whose period, $p$, is comparable to the signal wavelength, $\lambda$. A second type of metamaterials, the so-called effective media (i.e., metamaterials satisfying the condition $\lambda<<p$ ) are used in the present work. These metamaterials are divided into single negative media (SNG, i.e., effective media with negative magnetic permeability, $\mu<0$, or electric permittivity, $\varepsilon<0$ ) and double negative media (DNG, with simultaneous $\mu<0$ and $\varepsilon<0$ ), also called left-handed materials [11-13]. Physically, SNG can be implemented by using so called split-ring resonators (SRRs) [14] and their dual 
counterparts, the complementary split-ring resonators (CSRRs) [15]. Recently, CSRRs have been used as EMI reduction structures with a good behavior in terms of rejection band and signal integrity [16].

In this paper, a filter developed by means of effective media metamaterials based on CSRRs is used to reduce RF interference in a conventional differential amplifier based on an OPAMP. These kind of sub-wavelength resonators are able to achieve a good compromise between stop-band rejection level, cost, and PCB area consumption by taking into account that the design of the filter must cover the frequency separation between interference and useful signals. Specifically, the rejection frequency band has been designed at 2.4-GHz (Industrial, Scientific and Medical, ISM), due to the current multiple radiofrequency interference sources in this frequency range. The considered modulated frequency corresponds to $10 \mathrm{kHz}$. The paper is organized as follows: Section II describes the complementary split ring resonators geometry and its frequency behavior. Section III introduces the proposed layout (i.e., metamaterial filter combined with the differential amplifier) and the performed electromagnetic simulations. In Section IV, experimental results are discussed. Specifically, the filter attenuation, the demodulated signal reduction and the impact in terms of offset and modulation index have been analyzed. Finally, Section V summarizes the main conclusions of this work.

\section{COMPLEMENTARY SPLIT RING RESONATORS}

The sub-wavelength resonators used in this work in order to mitigate EMI at RF band are the CSRRs (the dual particle of the SRRs). Fig. 2 shows the basic topologies of both particles and their equivalent circuit models coupled to transmission lines. Essentially, SRRs (Fig. 2a) consist of a pair of metal rings etched on a dielectric slab with apertures in opposite sides which can be mainly excited by means of a parallel magnetic-field along its axis. If an array of SRRs is located close to a transmission line (i.e., a microstrip line), current loops can be induced in the rings and, at resonance, they reflect the incident host signal. Therefore they behave as an LC tank (described by $L_{S}$ and $C_{S}$ parameters) magnetically coupled (by using a mutual inductance, $M$ ) to the host line (defined by $L$ and $C$, Fig. 2b) [17]. From duality arguments based on an approximation of the Babinet's principle for dielectric boards, it is demonstrated that the CSRRs (Fig. 2c), the negative image of SRRs, roughly behave as their dual counterparts (i.e., their resonance frequency is approximately equal to that of the corresponding equivalent SRRs). Therefore, CSRRs are mainly excited by means of an axial time-varying electric field (magnetic field contribution is significantly minor) [18]. Since the main coupling contribution of these particles is electric, it can be basically described by means of a coupling capacitance $\left(C^{\prime}\right)$. Therefore, an efficient way to achieve stop-band frequency responses is to etch CSRRs in the ground plane of a structure such as a microstrip line (or similar) or even in the conductor strip. The synthesis of the filter is performed by using the LC equivalent model of CSRRs (described by $L_{C}$ and $C_{C}$ parameters) coupled by means of a capacitor (whose value, $C^{\prime}$, is slightly different to the corresponding to the capacitance of the transmission line) with the host line (Fig. 2d). According to the equivalent circuit model, the zero transmission frequency, $f_{Z}$, of each CSRR coupled to the victim line is given by,

$$
f_{Z}=\frac{1}{2 \pi \sqrt{L_{C} \cdot\left[C_{C}+C^{\prime}\right]}} .
$$

The main advantages of these sub-wavelength particles are their low implementation cost (no extra components or layers are required and they are implemented by means of conventional etching techniques) and the quasi-non-area consumption, since they are located in the ground plane. Moreover, key to the application of arrays of CSRRs (or SRRs and derived particles) to the synthesis of SNG is their small electrical size (periods involved are lower than $\lambda$ ) and, therefore, it implies an intrinsic compactness factor.

\section{Proposed Structure}

The test circuit consists of a conventional differential amplifier, as depicted in Fig. 3. The configuration corresponds to a basic instrumentation amplifier based on 4 resistors and one OPAMP. The resistors are chosen equal to provide an output voltage, $V_{o}$, approximately equal to the input voltage difference, $V_{o}=V_{I N+}-V_{I N-}$. In order to avoid RF EMI reaching the OPAMP input, a 4-CSRR filter has been designed and combined in the final prototype. Fig. 4 shows the PCB designed layout. As can be observed, two CSSR arrays have been located in the ground plane, underneath the input transmission lines carrying the signal of interest to the differential input, before it reaches the non linear part of the circuit. Notice that this distribution implies effective non-area consumption with respect to a conventional 1-layer design. Moreover, no extra lumped circuitry is needed and no series stage filter is required. Other CSRRs topologies, such as rectangular shape, can be used in order to compact the effective area of CSRRs with respect to the metal strip. Obviously, a trade-off between rejection level and number of stages appears. The design has been carried out by means of the Agilent ADS and Momentum software. The resonance frequency of the CSRRs has been designed in the vicinity of $2.4 \mathrm{GHz}$ in order to prevent susceptibility in the ISM band. The dimensions of the CSRRs have been calculated by developing full-wave electromagnetic simulations of single CSRRs coupled to a microstrip line section and a sweeping algorithm. The notch peak in the transmission frequency response reveals the presence of the CSRR. In order to compute the CSRRs dimensions, we determine $a$ and $b$ parameters to the minimum resolution of the used drilling machine $(200 \mu \mathrm{m})$. Concerning the initial computed frequency, it is possible to determine the dimensions of a circular SRR by following the algorithm detailed in [17]. According to theory the resonance frequency of the CSRR is approximately equal to the corresponding to its dual counterpart (SRR). Therefore, by considering the equivalent area of the circular CSRR we can compute an initial value for 
a square CSRR (by determining $c$ and $d$ dimensions). In fact, the involved single resonator dimensions have been slightly detuned in order to achieve a wider stop-band bandwidth (i.e., single CSRRs with close resonance frequencies). Therefore, a final simulation step based on a multiple tuning procedure has been developed. EMI filter simulations have been performed between external input ports (P1 and P3, Fig. 4) and internal ports (P2 and P4, Fig. 4) before OPAMP and circuitry stage (output port corresponds to P5, Fig. 4). CSRRs dimensions correspond to $a=b=0.2 \mathrm{~mm}$ for each stage, $c_{l}=d_{1}=6.7 \mathrm{~mm}$, $c_{2}=d_{2}=7.0 \mathrm{~mm}, \quad c_{3}=d_{3}=6.9 \mathrm{~mm}, \quad c_{4}=d_{4}=6.8 \mathrm{~mm}$, where $i=1,2,3,4$ corresponds to the stage number. The inter-resonator distance is $0.5 \mathrm{~mm}$. Electromagnetic simulations have been performed by considering a MC 100 FR4 substrate which will be described in next section.

Fig. 5a shows a detailed layout of the proposed 4-stage filter, whereas Fig. 5b depicts the corresponding full lumped circuit model by taking into account the electrical model illustrated in Fig. 2d. The inter-resonator coupling between adjacent CSRRs has been modelled by means of capacitances $C_{5}, C_{6}, C_{7}$. The extraction of the different parameters is directly related to the design methodology in order to implement these structures from some given specifications (typically the expected notch frequency, rejection level and the actual dimensions of the involved transmission line). The design methodology is summarized in Fig. 6 and consists of a sequence of steps described below. Since the model consists of several variables, the extraction of the parameters must be done sequentially. First, a single microstrip line is considered. The per-section inductance and capacitance of the line, $L$ and $C$, can be calculated from a transmission line calculator. The system is assumed lossless for simplicity (this is a reasonable assumption as a first step approximation). Second, the coupled $\mathrm{RF}$ interference frequency determines the dimensions of the CSRRs (their resonance frequency is determined by the $L_{C} C_{C}$ tank.). Basically, the CSRRs are electrically coupled to each microstrip line by means of the distributed capacitance, $C^{\prime}$. By analyzing a single CSRR coupled to the line (i.e., no interresonator coupling), two resonance frequencies arise: the frequency that nulls the shunt impedance (i.e., transmission zero frequency), given by (1) and the resonance frequency of the CSRR given by:

$$
f_{o}=\frac{1}{2 \pi \sqrt{L_{C} C_{C}}} .
$$

On the other hand, according to [19], the periodic structure under study by considering $\mathrm{C}^{\prime} \mathrm{A}=\mathrm{CM}=0$, satisfies (3),

$$
\cos \varphi=1+\frac{Z_{S}(j \omega)}{Z_{P}(j \omega)} .
$$

This equation allows the analysis of periodic circuits based on the cell depicted in Fig. 2(d) with the help of the dispersion relation and Bloch impedance. $\varphi$ denotes the phase shift of the elemental cell, and $Z_{P}$ and $Z_{S}$ correspond to the shunt and series impedance, respectively, of the T-circuit model. By using an electromagnetic simulator, it is possible to obtain the phase of the insertion loss transmittance, $\varphi_{S 21}(\omega)$. Since $Z_{P}$ and $Z_{S}$ are known expressions, by obtaining a given $\varphi_{S 2 l j}$ at its corresponding frequency, $\omega_{j}$, it is possible to solve the equation system (1-3) in order to determine each $L_{C}, C_{C}$ and $C$ '. Concerning the rejection level, the maximum number of subwavelength resonators guarantees the highest rejection level. Obviously, this fact implies a tradeoff with the available area and in case of close located CSRRs, the inter-resonator coupling must be taken into account in the model. Interresonator coupling is modeled by a mutual capacitance in a similar way to [20]. Therefore, by considering a second CSRR close to the original one, the inter-resonator coupling mutual capacitance, $C_{M}$, can be extracted as a fitting parameter. Table I summarizes the values of the parameters included in the equivalent model depicted in Fig. 5b.

TABLE I

EQUIVALENT CIRCUIT MODEL PARAMETERS

\begin{tabular}{cc}
\hline \hline Component & Value \\
\hline L1, L2, L3, L4, L5, & $1.5907 \mathrm{nH}$ \\
L6, L7, L8 & \\
Lc1 & $0.116 \mathrm{nH}$ \\
Lc2 & $0.112 \mathrm{nH}$ \\
Lc3 & $0.122 \mathrm{nH}$ \\
Lc4 & $0.147 \mathrm{nH}$ \\
C1 & $3.887 \mathrm{pF}$ \\
C2 & $4 \mathrm{pF}$ \\
C3 & $3.846 \mathrm{pF}$ \\
C4 & $2.188 \mathrm{pF}$ \\
C5, C6, C7 & $0.05 \mathrm{pF}$ \\
Cc1 & $30.096 \mathrm{pF}$ \\
Cc2 & $33.136 \mathrm{pF}$ \\
Cc3 & $33.130 \mathrm{pF}$ \\
Cc4 & $33.798 \mathrm{pF}$ \\
Term1, Term2 & $50 \Omega$ \\
\hline \hline
\end{tabular}

Fig. $7 \mathrm{a}$ illustrates the electromagnetic simulation corresponding to the discussed filter in comparison with the single frequency response of each CSRR. It is observed a significant enhancement in the bandwidth and rejection level by including the 4-stages together due to the coupling between them. Moreover, Fig. $7 \mathrm{~b}$ shows the detailed fitting between the proposed equivalent circuit frequency response and the EM simulation. Fig. 8a depicts the CSRRs filter electromagnetic response by considering the overall implementation. As can be observed, a significant rejection level is obtained $(|\mathrm{S} 21|<-40$ dB) at the frequency band of interest with an enhanced bandwidth with respect to a peak notch response. The enlargement of the operational bandwidth concerning to the case of CSRRs with the same dimensions depends on the number of used stages. Obviously, the rejection level can be increased by adding extra CSRRs stages and electric coupling, but a trade-off exists with respect to the dimensions of input transmission lines. Regarding the impact of PCB tolerances on filtering characteristics, the dielectric constant tolerance $\left(\varepsilon_{r}=4.5 \pm 0.1\right)$ on the central frequency and rejection level corresponds to a variation of $0.8 \%$ and $1 \mathrm{~dB}$, respectively, whereas the corresponding to the drilling machine resolution $( \pm 0.01 \mathrm{~mm}$, typical for the PCB manufacturing) corresponds 
to $1 \%$ and $1.5 \mathrm{~dB}$, respectively. Fig. $8 \mathrm{~b}$ shows the group delay of the proposed filter in terms of frequency. As can be observed, the maximum value corresponds to $12 \mathrm{~ns}$ at a frequency close to $2.3 \mathrm{GHz}$.

\section{EXPERIMENTAL RESULTS}

Two experimental prototypes have been fabricated and tested. Both present the same top level metal layer layout and are differentiated by a conventional ground plane in the first case and a ground plane disturbed by etched CSRR arrays in the second. Fig. 9 shows the prototype setup consisting of a 3port 4-stage CSRR loaded parallel transmission lines, which have been designed to obtain a stop band filter around 2.4 GHz. The OPAMP used in the prototypes is a $U A 741 C D$, supplied by two voltage regulators, a MC78M15BDTG (15V) and a $M C 79 M 15 C D T G(-15 \mathrm{~V})$. All resistor values are $1 \mathrm{k} \Omega$. Decoupling capacitors $(100 \mathrm{nF}$ and $10 \mu \mathrm{F})$ have been also used for the supply lines. The substrate corresponds to the commercial MC $100 F R 4$ (dielectric constant $\varepsilon_{\mathrm{r}}=4.5$, thickness $h=1.53 \mathrm{~mm}$ ), whereas metal layers correspond to copper (thickness, $t=35 \mu \mathrm{m}$ ). Specifically, $50 \Omega$ microstrip access lines are considered with dimensions: width $W=2.84 \mathrm{~mm}$, length $l=4 \mathrm{~cm}$, and separation $s=6.55 \mathrm{~mm}$. The CSRRs dimensions (detailed in the previous section) have been etched by means of a LPKF S62 drilling machine. The total circuit area is $6.9 \times 4.8 \mathrm{~cm}^{2}$.

The test prototypes have been measured by means of a Rohde \& Schwarz ESPI spectrum analyzer and Tektronix TDS $510 \mathrm{~A}$ oscilloscope. The test setup consists of emulating the RF EMI coupling by means of a signal modulated in AM (whose carrier frequency is $f_{C}=2.4 \mathrm{GHz}$ ) with a low frequency sinus signal (whose modulated frequency has been chosen as $f_{m}=10$ $\mathrm{kHz}$ ). The direct power injection carrier amplitude, $A_{C}$, corresponds to $A_{C}=-10 \mathrm{dBm}$ and the modulation index, $m$, is $m=50 \%$. Fig. 10 illustrates the described experimental setup. Fig. 11 shows the comparison between the circuit output modulated spectrum including or not the CSRRs filter. As can be seen, the intrinsic frequency response of the OPAMP at high frequency implies an attenuation of $-25 \mathrm{~dB}$ for the conventional structure. By using the CSRRs implementation the attenuation is improved significantly, and a rejection level higher than $20 \mathrm{~dB}$ is achieved. The experimental output spectrum at the low operation frequency $(10 \mathrm{kHz})$ reveals a disturbance on the order of $20 \mathrm{~dB}$ for the conventional case (Fig. 12), which is produced by the non-linear behavior of OPAMP, as explained in Section I. However, the CSRRs prototype completely removes this EMI effect, since the impact of resonators notably filters the undesired noise signal at this frequency. In order to evaluate the effectiveness of the proposed implementation, several parameters have been tested. Fig. 13 illustrates the experimental DC offset voltage in terms of interference amplitude. When the noise signal is injected, a significant increase of the offset with EMI amplitudes higher than $-5 \mathrm{dBm}$ is observed in the non-filtered circuit, whereas in the same conditions, the offset of the prototype equipped with the CSRR filter remains constant ($1.19 \mathrm{mV})$. Notice also that the offset level is almost 4 times lower in CSRR filtered prototype, since the best offset level for the conventional device is approximately $-4 \mathrm{mV}$. Fig. 14 shows the impact of the modulation index on the output level, $V_{o}$, at frequency of interest $(10 \mathrm{kHz})$ for both prototypes. By fixing $A_{C}=-5 \mathrm{dBm}$, the $m$ sweep again reveals a constant behavior for the CSRRs implementation $\left(V_{o}=-90 \mathrm{dBm}\right)$ whereas the conventional one dramatically increases the output level of the disturbance. $V_{o}$ has also been tested by fixing $m=50 \%$ and sweeping the EMI voltage level, $A_{C}$ (Fig. 15). In this case the differences are produced from $A_{C}>-20$ $\mathrm{dBm}$. Obviously, at high interference voltage levels, the total level rejection of the filter is highlighted $(45 \mathrm{~dB})$ in good agreement with the simulation results (Fig. 8a).

Therefore, it is demonstrated that, for high frequency, CSRRs can be a good solution to reduce EMI susceptibility in circuits based on OPAMPs. In fact, this method implies quasinon-cost, since CSRRs can be implemented by conventional etching process in the ground plane, and no extra area or electronic devices are required in comparison with conventional EMI filters or shielding solutions.

\section{CONCLUSIONS}

In summary, it has been demonstrated that EMI effects due to random RF noise signals reaching the OPAMP input circuits, which present an inherent non-linear behavior, can be significantly reduced by means of filters based on CSRR. Basically, the demodulated low frequency signal is attenuated by these conveniently tuned sub-wavelength resonators. A significant increase of device RF immunity as well as DC offset minimization has been demonstrated, both by simulation and experimentally. In fact, ground loaded CSRR transmission lines can be a compact-low-cost method in order to significantly decrease the PCB RF coupling interference at the ISM band. Simulated and experimental results show a 45 $\mathrm{dB}$ coupling reduction at $2.4 \mathrm{GHz}$. The authors are confident about the application of these structures for EMI reduction in planar electronic circuits operating at high frequencies/datatransmission rates.

\section{REFERENCES}

[1] C.E. Larson, J. M. Roe, "A modified Ebers-Moll transistor model for RF-interference analysis", IEEE Transactions on Electromagnetic Compatibility, vol. EMC-21, pp. 283-290, November 1979.

[2] J. Gago, J. Balcells, D. González, M. Lamich, J. Mon, A. Santolaria, "EMI susceptibility model of signal conditioning circuits based on operational amplifiers", IEEE Transactions on Electromagnetic Compatibility, vol. 49, pp. 849-859, November 2007.

[3] F. Fiori, P.S. Crovetti, "Complementary differential pair with high immunity to RFI", Electronics Letters, vol. 38, pp. 1663-1664, December 2002.

[4] F. Zhang, C. Li, "Wide stopband lowpass filter with a novel U-shaped ground aperture"; International Journal of Electronics, vol. 94, pp. 617621, 2007.

[5] G. Lovat, S. Celozzi, "Shielding effectiveness of planar negativepermeability screens", IEEE International Symposium of Electromagnetic Compatibility, pp. 1-6, 2008.

[6] S. Shahparnia and O. M. Ramahi, "Electromagnetic interference (EMI) reduction from printed circuit boards (PCB) using electromagnetic bandgap structures", IEEE Transactions on Electromagnetic Compatibility, vol. 46, pp. 580-587, November 2004. 
[7] T.-L. Wu, Y.-H. Lin, T.-K. Wang, C.-C. Wang and S.-T. Chen, "Electromagnetic bandgap power/ground planes for wideband suppression of ground bounce noise and radiated emission in high-speed circuits", IEEE Transactions on Microwave Theory and Techniques, vol. 53, pp. 2935-2942, September 2005.

[8] S.-H. Joo, D.-Y. Kim and H.-Y. Lee, "A S-bridged inductive electromagnetic bandgap power plane for suppression of ground bounce noise", IEEE Microwave and Wireless Components Letters, vol. 17, pp. 709-711, October 2007.

[9] B. M.-Iravani and O. M. Ramahi, "Design, implementation and testing of miniaturized electromagnetic bandgap structures for broadband switching noise mitigation in high-speed PCBs", IEEE Transactions on Advanced Packaging, vol. 30, pp. 171-179, May 2007.

[10] B. M.-Iravani and O. M. Ramahi, "Suppression of EMI and electromagnetic noise in packages using embedded capacitance and miniaturized electromagnetic bandgap structures with high-k dielectrics", IEEE Transactions on Advanced Packaging, vol. 30, pp. 776-788, November 2007.

[11] V.G. Veselago, "The electrodynamics of substances with simultaneously negative values of $\varepsilon$ and $\mu$ ", Sov. Phys.-Usp., vol. 10, pp. 509-514, Jan.Feb. 1968.

[12] D.R. Smith, W.J. Padilla, D.C. Vier, S.C. Nemat-Nasser and S. Schultz, "Composite medium with simultaneously negative permeability and permittivity", Physical Review Letters, vol. 84, pp. 4184-4187, May 2000.

[13] G.V. Eleftheriades, A.K. Iyer and P.C. Kremer, "Planar negative refractive index media using periodically $L-C$ loaded transmission lines", IEEE Transactions on Microwave Theory and Techniques, vol. 50, pp. 2702-2712, December 2002.

[14] J.B. Pendry, A. J. Holden, D. J. Robbins, and W. J. Stewart, "Magnetism from conductors and enhanced nonlinear phenomena", IEEE Transactions on Microwave Theory and Techniques, vol. 47, pp. 20752084, November 1999.

[15] F. Falcone, T. Lopetegi, J.D. Baena, E. Marqués, F. Martín and M. Sorolla, "Effective negative- $\varepsilon$ stop-band microstrip lines based on complementary split ring resonators", IEEE Microwave and Wireless Component Letters, vol. 14, pp. 280-282, June 2004.

[16] I. Gil and R. Fernández, "Comparison between complementary split ring resonators and electromagnetic band-gap as EMI reduction structures", International Symposium on EMC Europe 2010, Wroclaw, Poland, September 2010.

[17] J.D. Baena, J. Bonache, F. Martín, R. Marqués, F. Falcone, T. Lopetegui, M.A.G. Laso, J. García-García, I. Gil, M. Flores Portillo and M. Sorolla. "Equivalent-circuit models for split-ring resonators and complementary split-ring resonators coupled to planar transmission lines", IEEE Transactions on Microwave Theory and Techniques, vol. 53, pp. 1451-1461, April 2005.

[18] R. Marqués, F. Medina, and R. R.-E. Idrissi, "Role of bianisotropy in negative permeability and left handed metamaterials," Phys. Rev. B, Condens.Matter, vol. 65, pp. 144 441-144 446, Apr. 2002.

[19] D.M. Pozar, "Microwave Engineering", Addison-Wesley, 1990.

[20] I. Gil, J. Bonache, M. Gil, J. García-García, F. Martín and R. Marqués, "Accurate circuit analysis of resonant-type left handed transmission lines with inter-resonator coupling", Journal of Applied Physics, vol. 100, pp. 074908-1-10, October 2006.

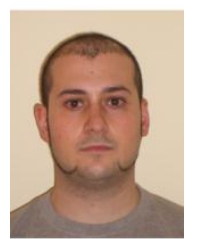

Daniel Pérez was born in Barcelona, Spain, in 1979. He received the Industrial Electronics and Automatic Control Engineering degree from the Universitat Politècnica de Catalunya, Barcelona, Spain, in 2008, and is currently working toward the Ph.D. degree at the Universitat Politècnica de Catalunya. He is also working as engineer with the Universitat Politècnica de Catalunya. His research interests include EMC and microwave devices and metamaterials.

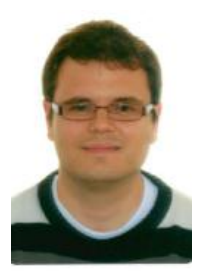

Ignacio Gil (S’05-M'07) was born in 1978 in Barcelona, Spain. He received degrees in physics and electronics engineering in 2000 and 2003, and then his PhD in 2007 from the Universitat Autònoma de Barcelona, Spain.

From 2003 until 2008 he was Assistant Lecturer in Electronics and a researcher with the RF-Microwave Group in the Electronics Engineering Department, Universitat Autonòma de Barcelona, Spain. From 2006 until 2008 he worked for EPSON Europe Electronics $\mathrm{GmbH}$ where he developed high-performance integrated RF CMOS circuits, transceivers and system design. In 2008 he joined the Electronics Engineering Department, Universitat Politècnica de Catalunya, Spain as lecturer and researcher. In recent years, he has been involved in different research activities including passive and active RF and microwave devices and circuits, metamaterials and EMC.

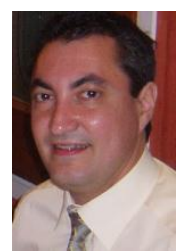

Javier Gago was born in Barcelona in 1965 and received the Master Engineering and Doctoral degree fron the Universitat Politècnica de Catalunya in 1991 and 2003, respectively. From 1993 to 2007 he is assistant professor in the Electronics Engineering Dept. At present he is associate professor in the same Department. His topics of interest are electromagnetic compatibility in industrial electronics, EMI susceptibility on electronic components, HF modelling of switched converters, power conversion and instrumentation. $\mathrm{He}$ is author or co-author of more than 20 technical papers published in international journals and conferences.

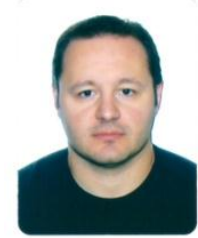

Raúl Fernández (M’06) was born in 1975 in Barcelona, Spain. He received degrees in telecommunications and electronics engineering in 1997 and 1999, and then his $\mathrm{PhD}$ in 2007 from the Universitat Autònoma de Barcelona, Spain. From 1998 until 2001 he worked for SONY España as an RF Engineer, where he developed analog and digital TV tuners. From 2001 until 2007 he was part time Assistant Lecturer in Electronics in the Electronics Engineering Department, Universitat Autonòma de Barcelona, Spain. In 2008 he joined the Electronics Engineering Department, Universitat Politècnica de Catalunya, Spain as a lecturer and researcher. In recent years, he has been involved in different research activities in the CMOS reliability field. Currently, he is involved on EMC robustness of integrated circuits.

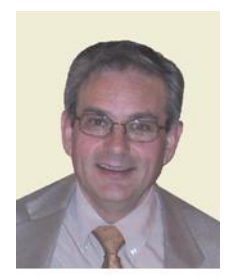

Josep Balcells, was born in Spain, in 1949. He received the Master and PhD. Degrees in Industrial Engineering from the Universitat Politècnica de Catalunya (UPC), Spain, in 1975 and 1983, respectively.

From 1975 to 1986, he was Associate Professor with the Department of Electronics Engineering, UPC. From 1978 to 1986 , he was the head of R\&D in Power Electronics in AGUT SA (now a GE-group company). Since 1986 he is Professor at the Electronics Engineering Department of UPC, and technical consultant of CIRCUTOR SA. His research interests include EMC in power systems, renewable energy systems, and design of power converters. He is author of several books and many papers related with EMC in power systems, power quality measurement and filtering and he has been responsible for several research projects funded by the Spanish Ministry of Science and Technology, Ministry of Industry and by the European Union in such topics.

Dr. Balcells is a Senior Member of IEEE and is serving as an Associate Editor of the IEEE TRANSACTIONS ON INDUSTRIAL ELECTRONICS since 2004. 


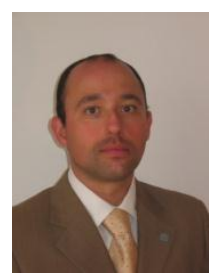

David González received the Master Engineering and Doctoral degree from the Universitat Politècnica de Catalunya in 1993 and 2001, respectively. From 1995 to 2005 he was assistant professor in the Electronics Engineering Department. At present he is associate professor in the same Department. His topics of interest are electromagnetic compatibility in industrial electronics, HF modelling of switched power converters, power conversion and power quality. He serves regularly as reviewer of several IEEE Transactions and IEEE sponsored conferences. $\mathrm{He}$ is author or co-author of more than 30 technical papers published in international journals and conferences.

Néstor Berbel was born in Barcelona, Spain, in 1979. He received the M.S. degree in Electronics from the Universitat Politècnica de CAtalunya (UPC), Barcelona, Spain in 2004. Since 2003, he has been an Assistant Professor, in the Department of Electronics Engineering, UPC, where he is working toward the Doctorate degree in Electronic Engineering. His current research interests include EMC robustness of integrated circuits.

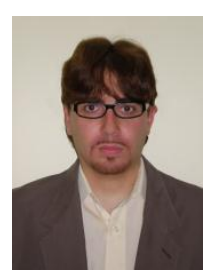

Juan Mon was born in Galicia, Spain in 1977. He received the M.S degree in telecommunications from the Universitat Politècnica de Catalunya in 2005.

From 2000 through 2002, he worked for Marconi Iberia S.A. Since 2003, he has been an Assistant Professor in the Electronics Engineering Department. Now $\mathrm{He}$ is attending the doctorate degree in electronic engineering from the Universitat Politècnica de compatibility in power converters. 


\section{Figure Captions}

Fig. 1. Response of linear ICs to EMI at the input. (a) EMI source. (b) OPAMP response.

Fig. 2. (a) Topology of the SRR with its relevant dimensions, and (b) lumped-element equivalent circuit for the unit-cell of the SRR coupled to a transmission line. (c) Topology of the CSRR with its relevant dimensions. Metallization zones are depicted in grey. (d) Lumped-element equivalent circuit of the CSRR coupled to a transmission line.

Fig. 3. Schematic of the test circuit.

Fig. 4. Designed layout. The black layer corresponds to the up side metal, whereas the grey layer corresponds to ground, where CSRRs have been etched.

Fig. 5. (a) Layout detail of the implemented filter. (b) Equivalent circuit model.

Fig. 6. Design methodology synthesis.

Fig. 7. (a) Electromagnetic simulation of the proposed 4-stage filter in comparison with the single frequency response of each CSRR. (b) Equivalent circuit frequency response (black) and electromagnetic simulation (green).

Fig. 8. (a) Full-wave electromagnetic simulation Fabricated 4port prototype device. Insertion losses, $|\mathrm{S} 21|$, and return losses, IS11I, are depicted. (b) Group delay of the proposed filter vs frequency.

Fig. 9. Fabricated 3-port prototype device. (a) Top side. (b) Bottom side including CSRRs.

Fig. 10. Experimental setup.

Fig. 11. Experimental AM modulation output device spectrum for conventional and CSRRs prototypes.

Fig. 12. Experimental demodulation output device spectrum for conventional and CSRRs prototypes.

Fig. 13. Experimental DC offset vs. RF interference amplitude for conventional and CSRRs prototypes $(m=50 \%)$.

Fig. 14. Experimental output level vs. modulation index at the interest operation frequency of both prototypes $\left(A_{C}=-5 \mathrm{dBm}\right)$.

Fig. 15. Experimental output level vs. EMI voltage level at the interest operation frequency of both prototypes $(m=50 \%)$. 
Fig. 1

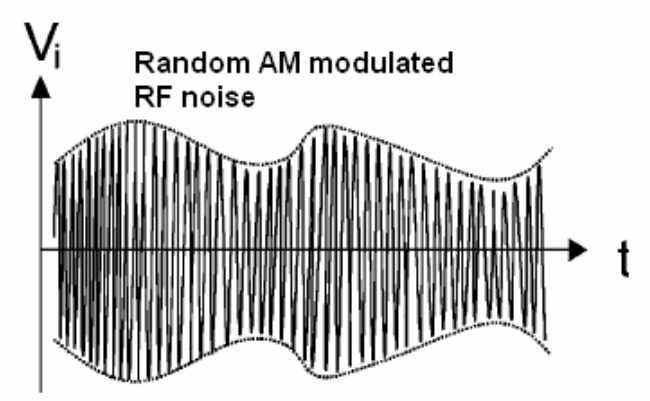

a)

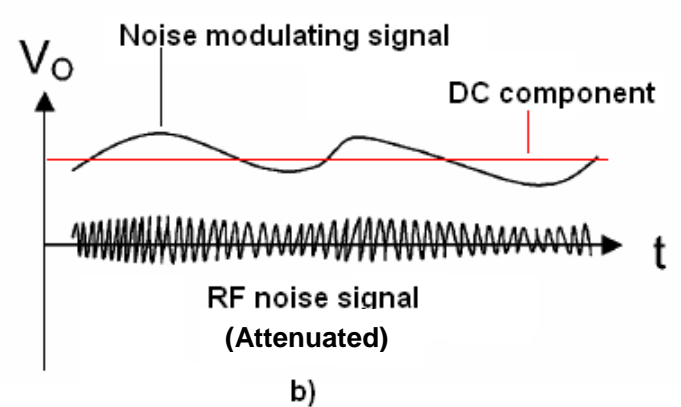

b) 
Fig. 2

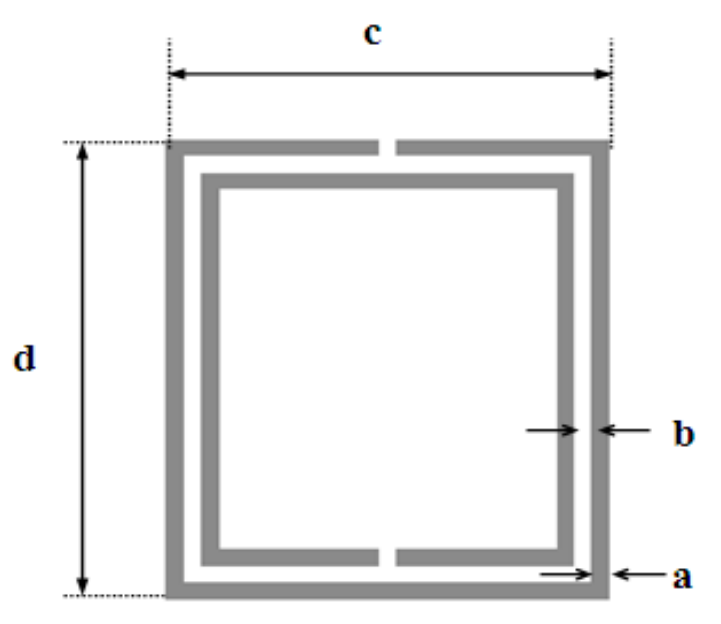

(a)

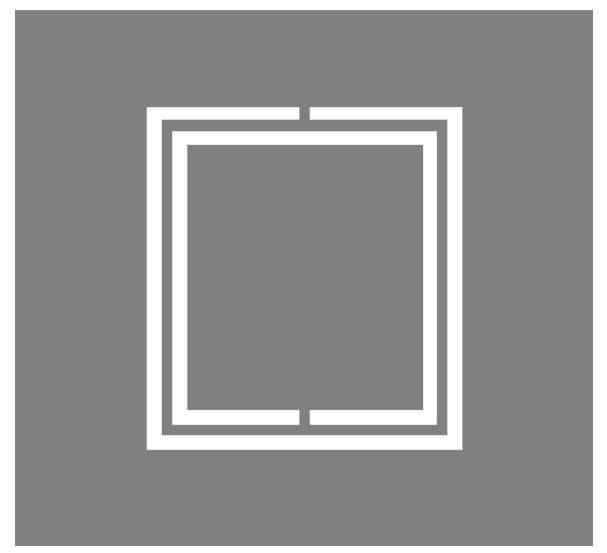

(c)

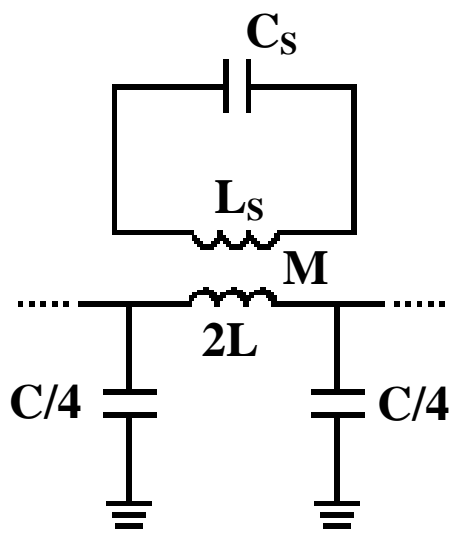

(b)

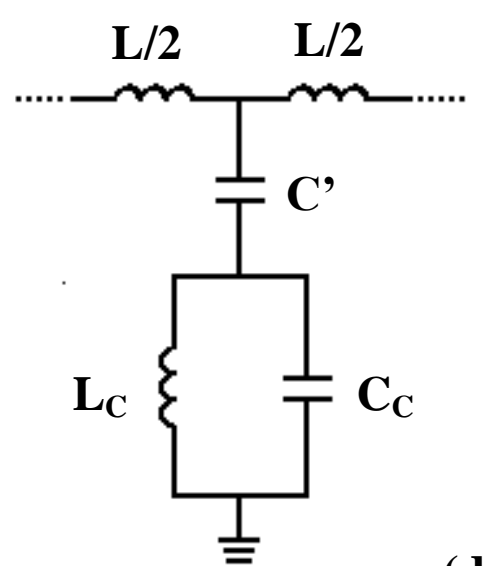

(d) 
Fig. 3

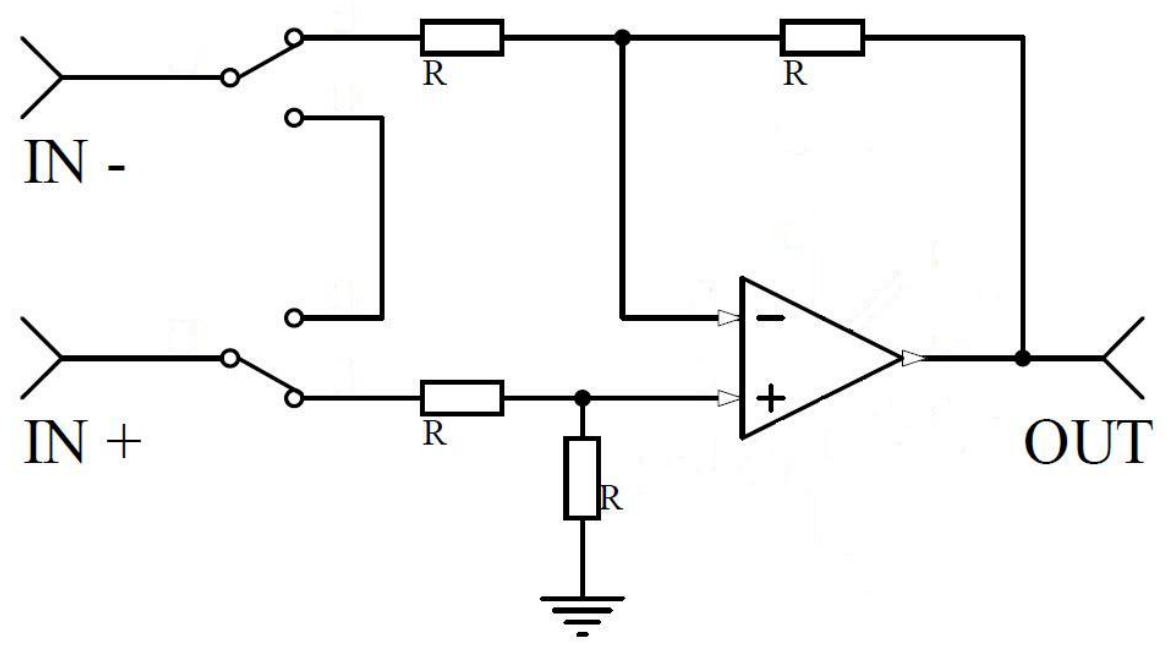


Fig. 4

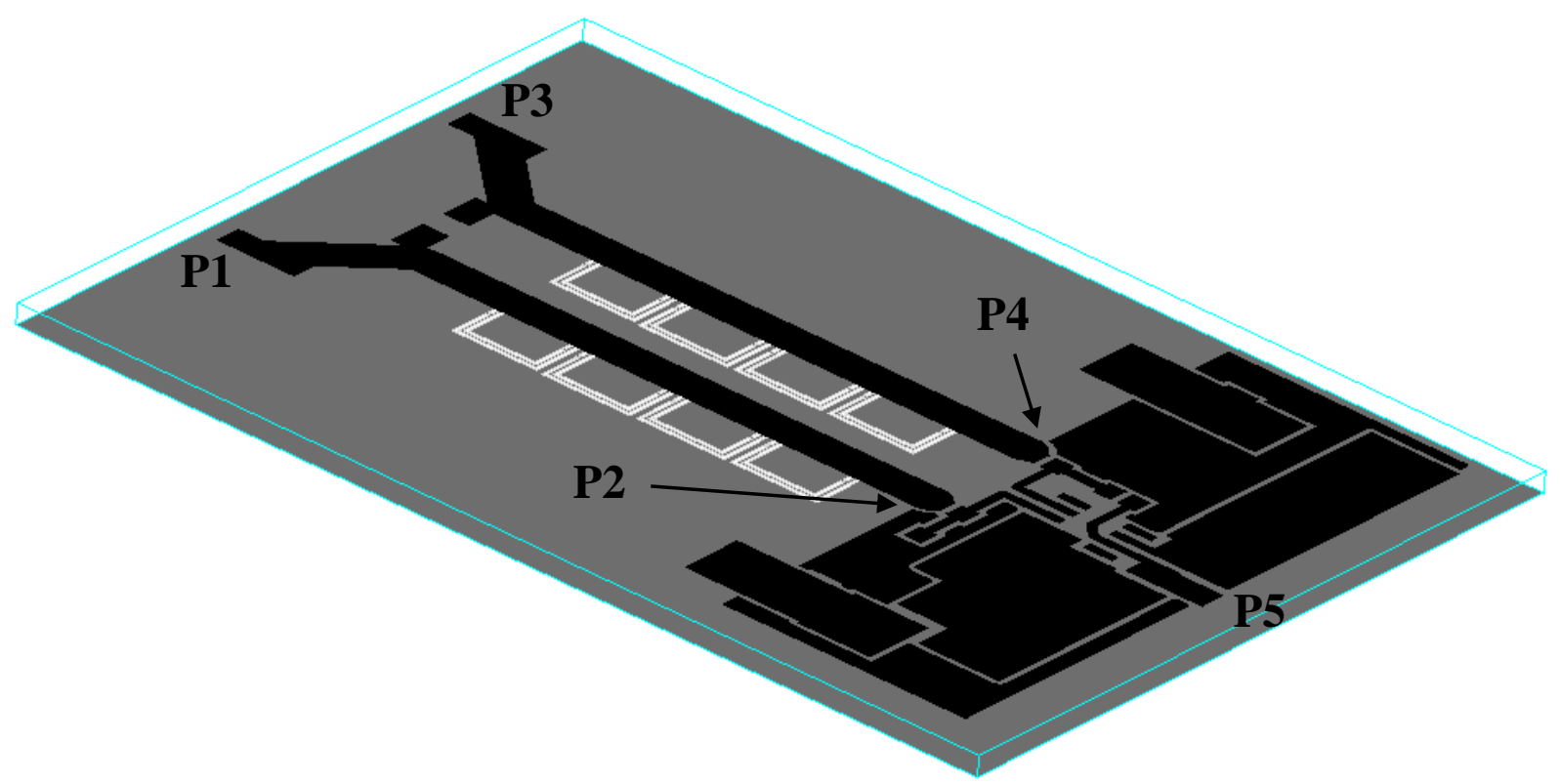


Fig. 5

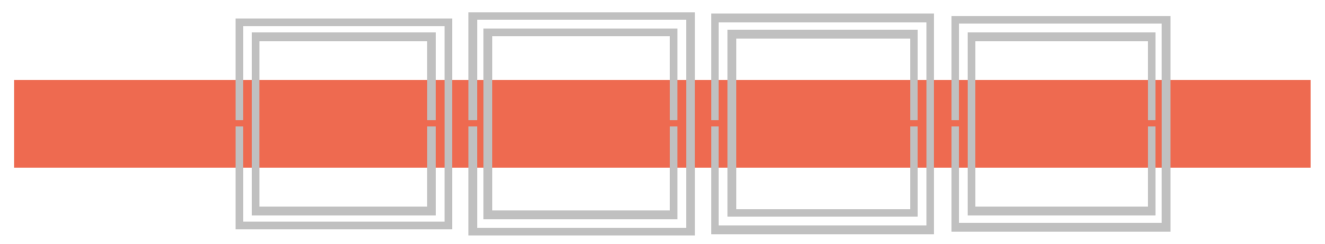

(a)

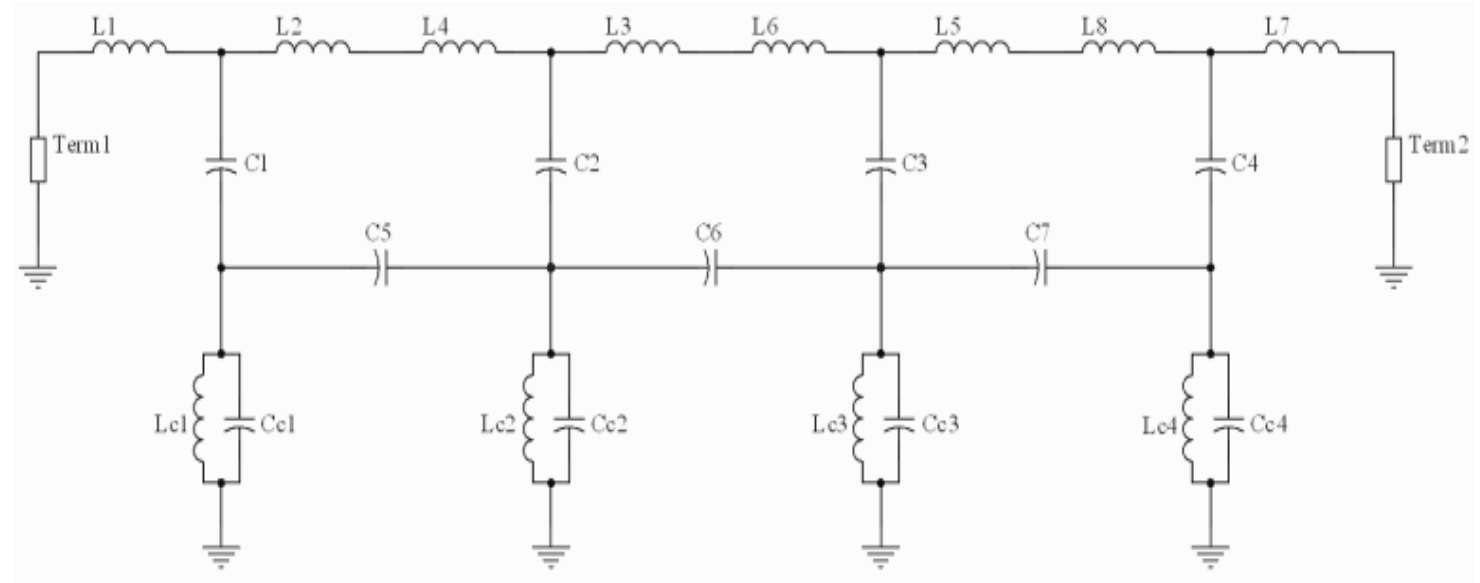

(b) 


\section{Fig. 6}

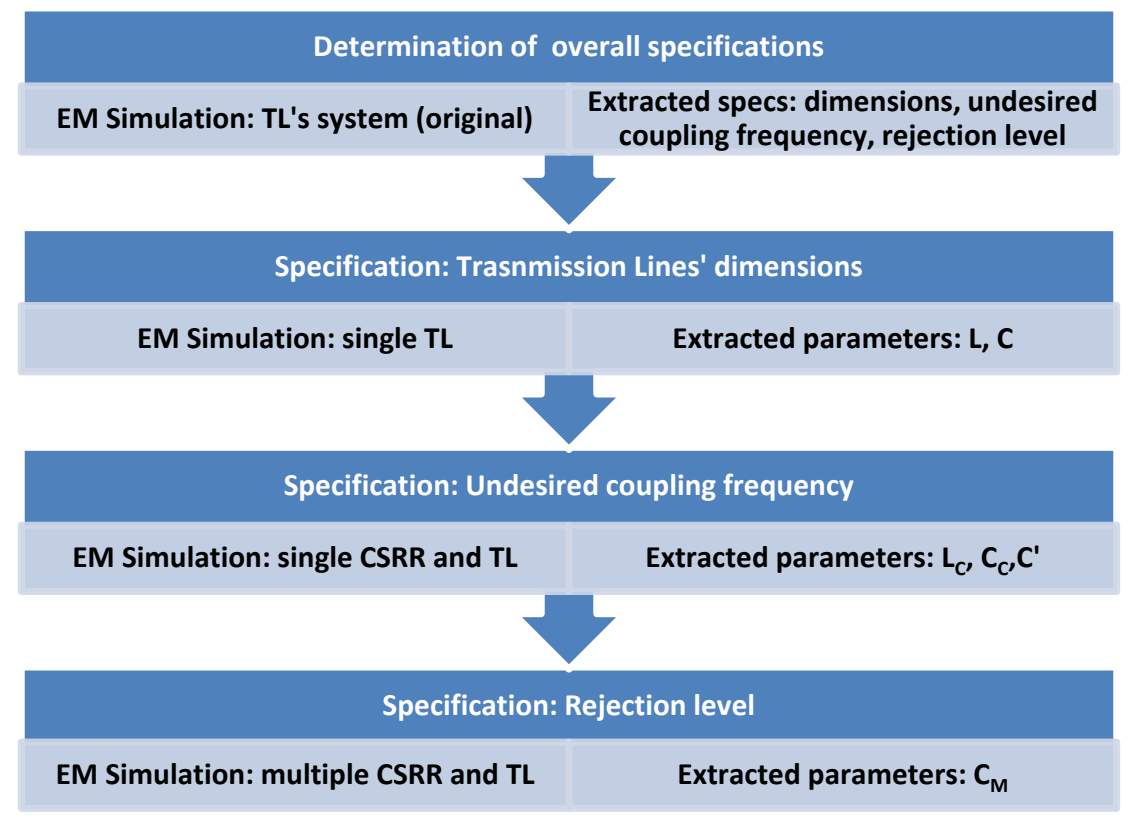


Fig. 7
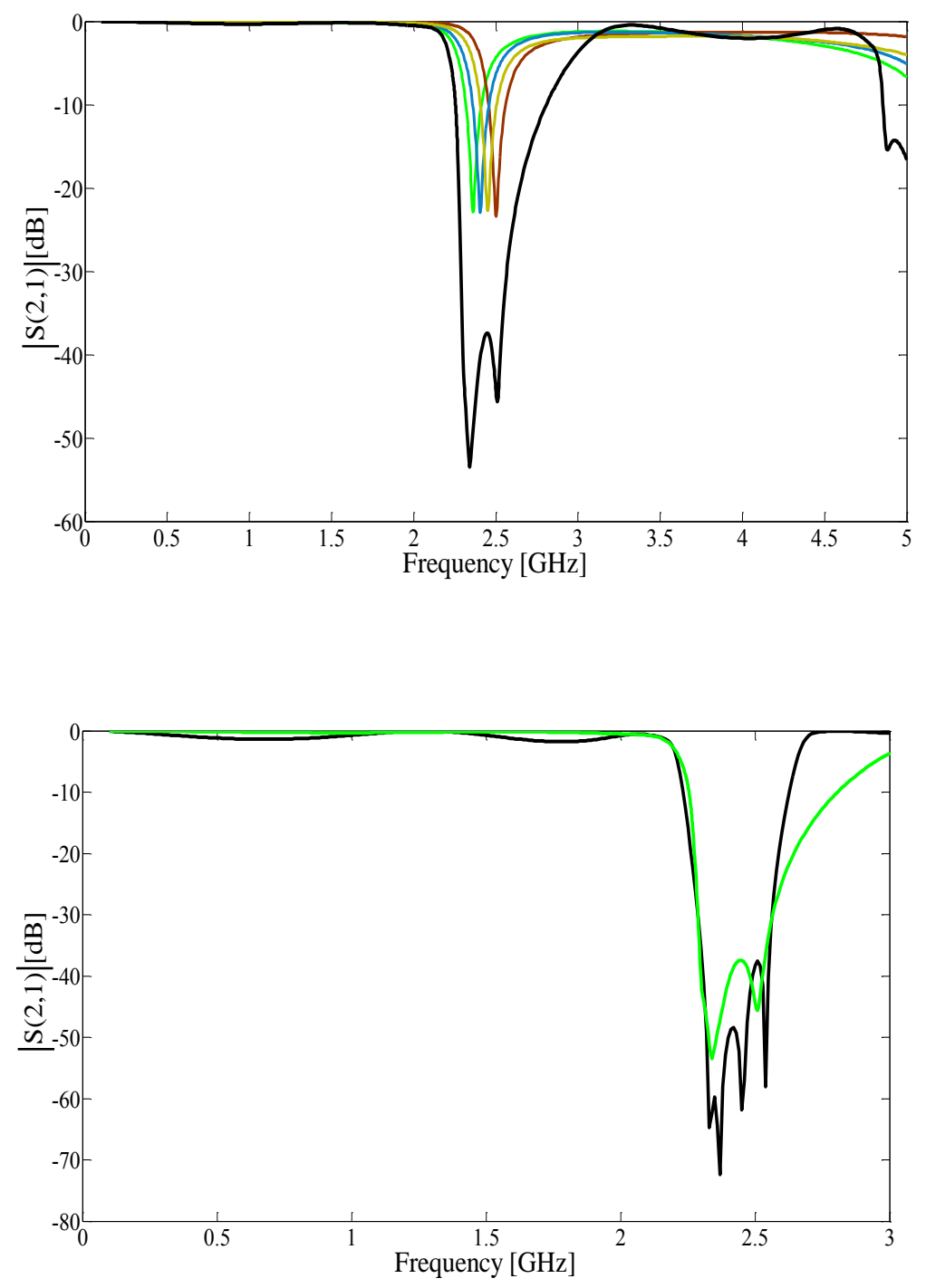
Fig. 8
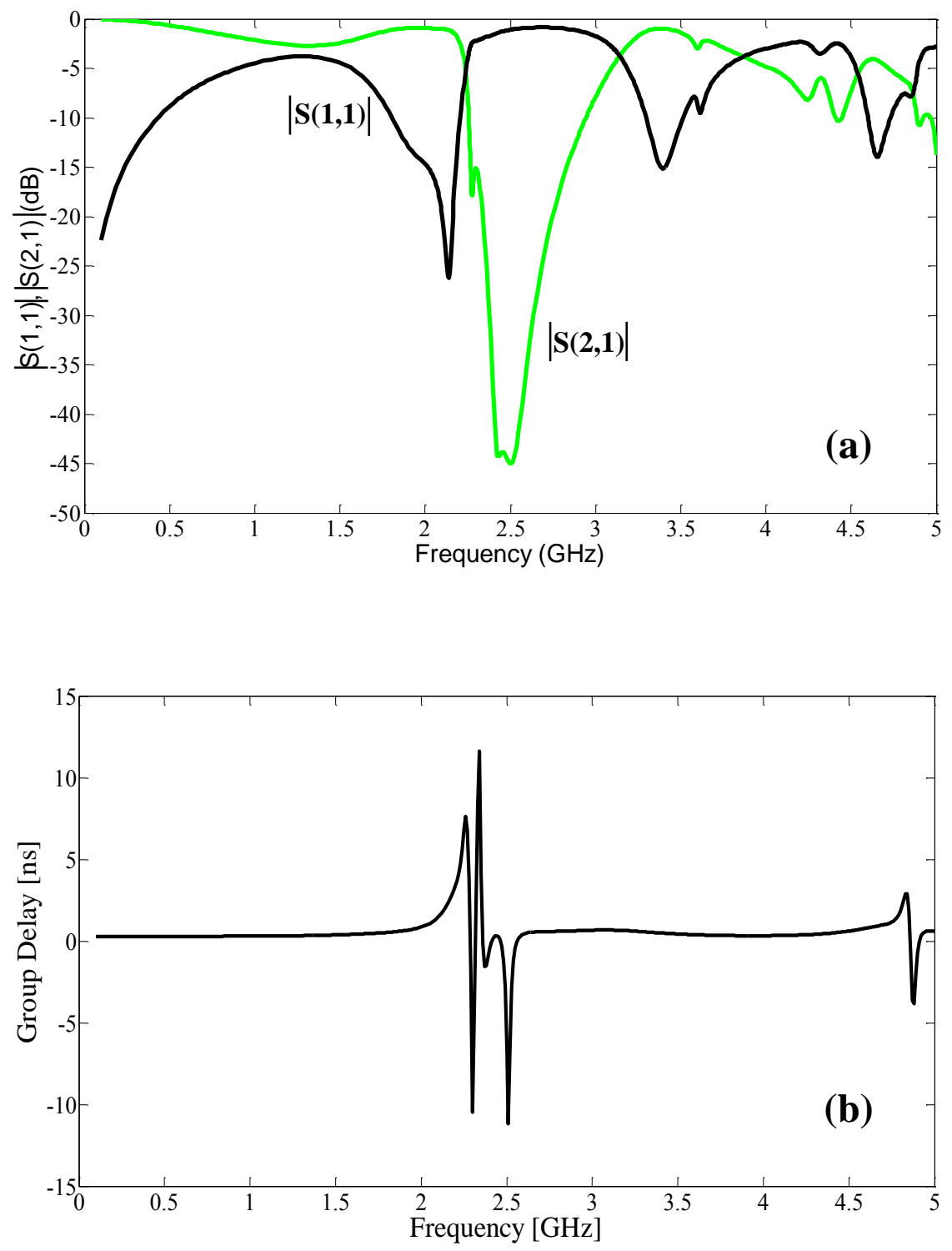
Fig. 9
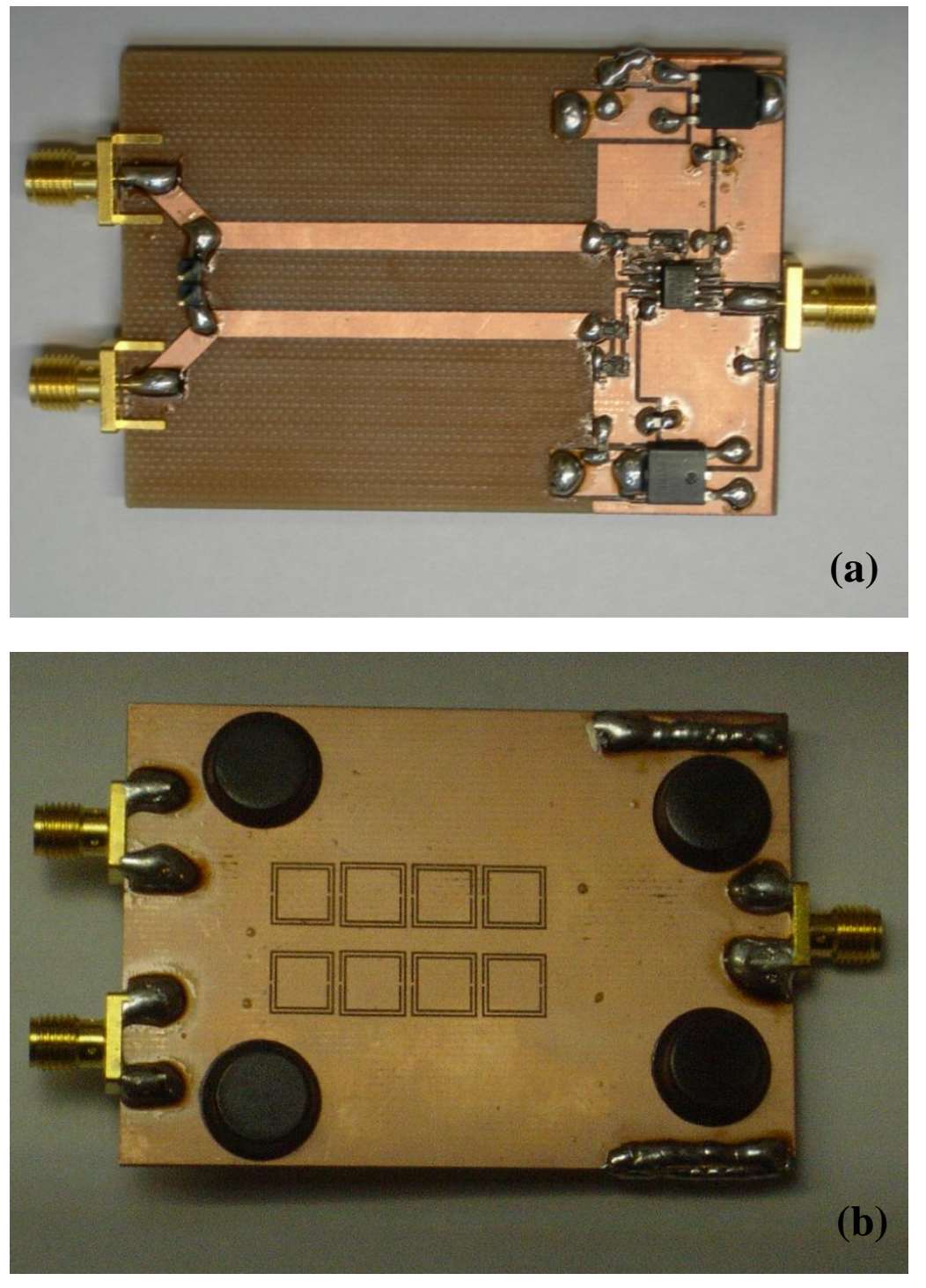
Fig. 10

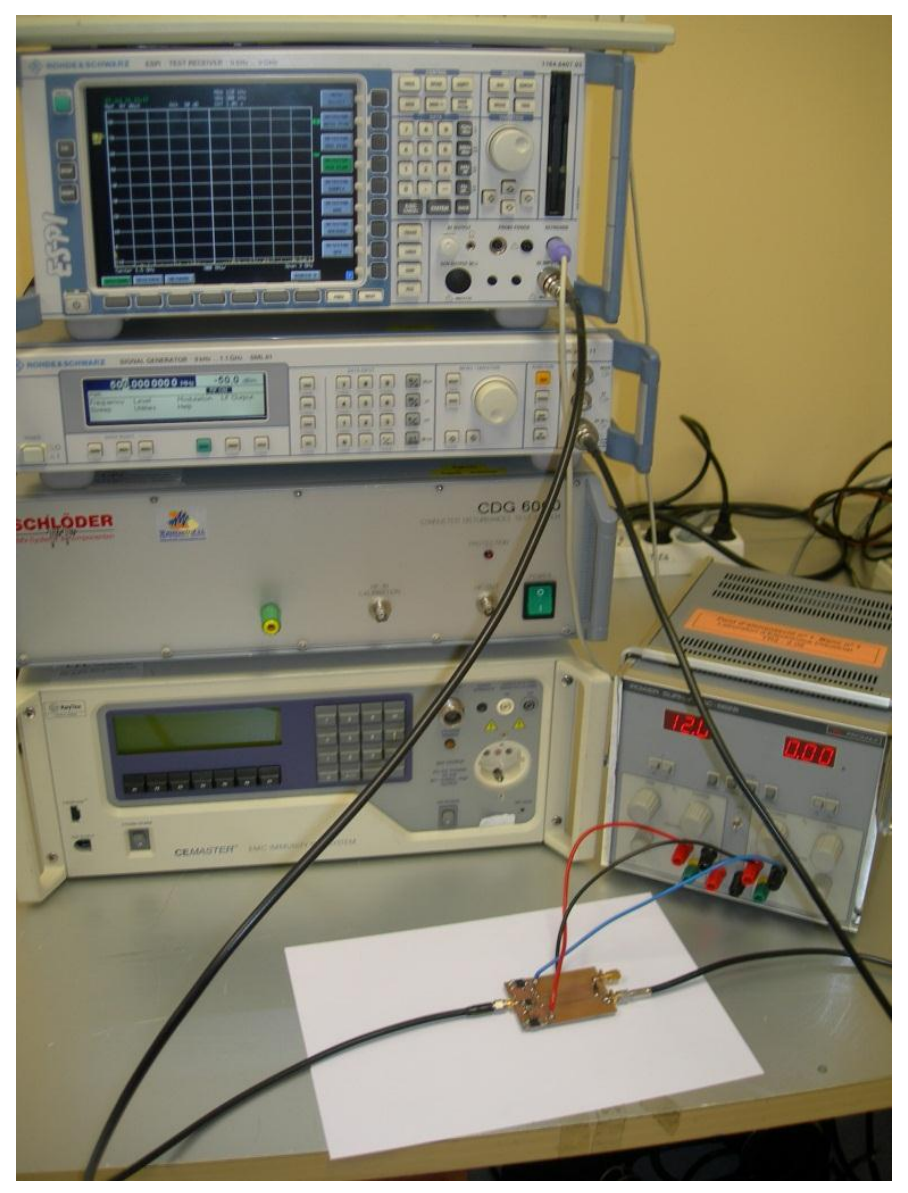


Fig. 11

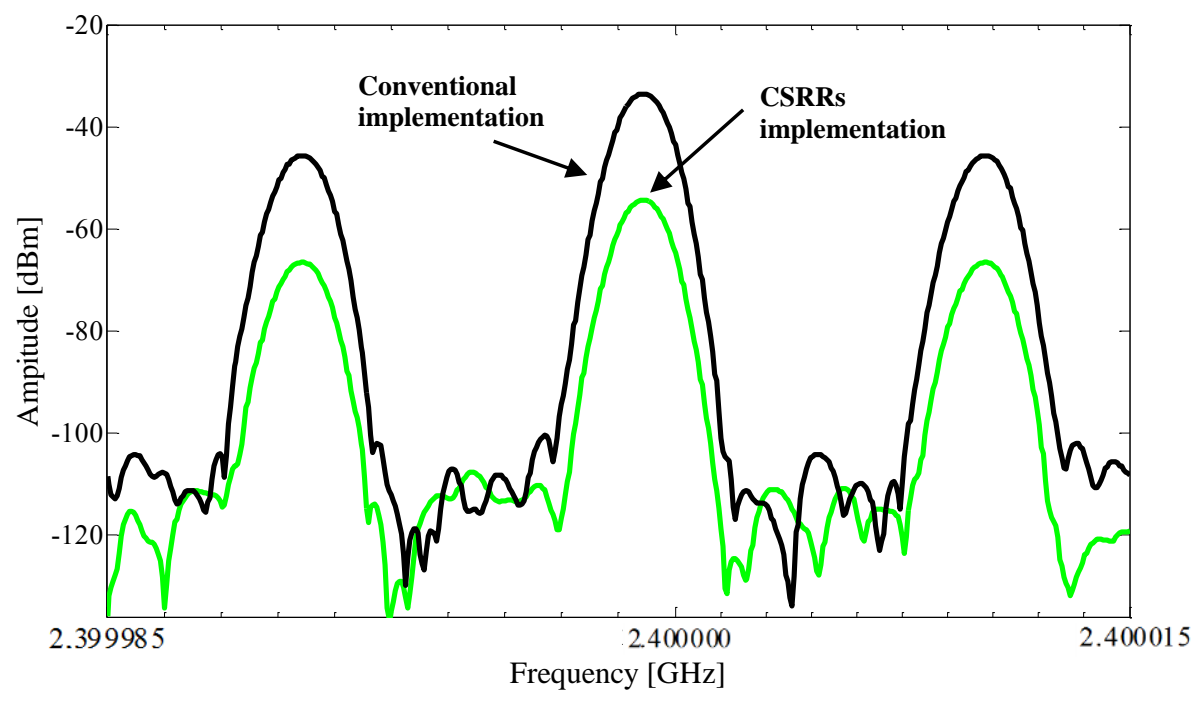


Fig. 12

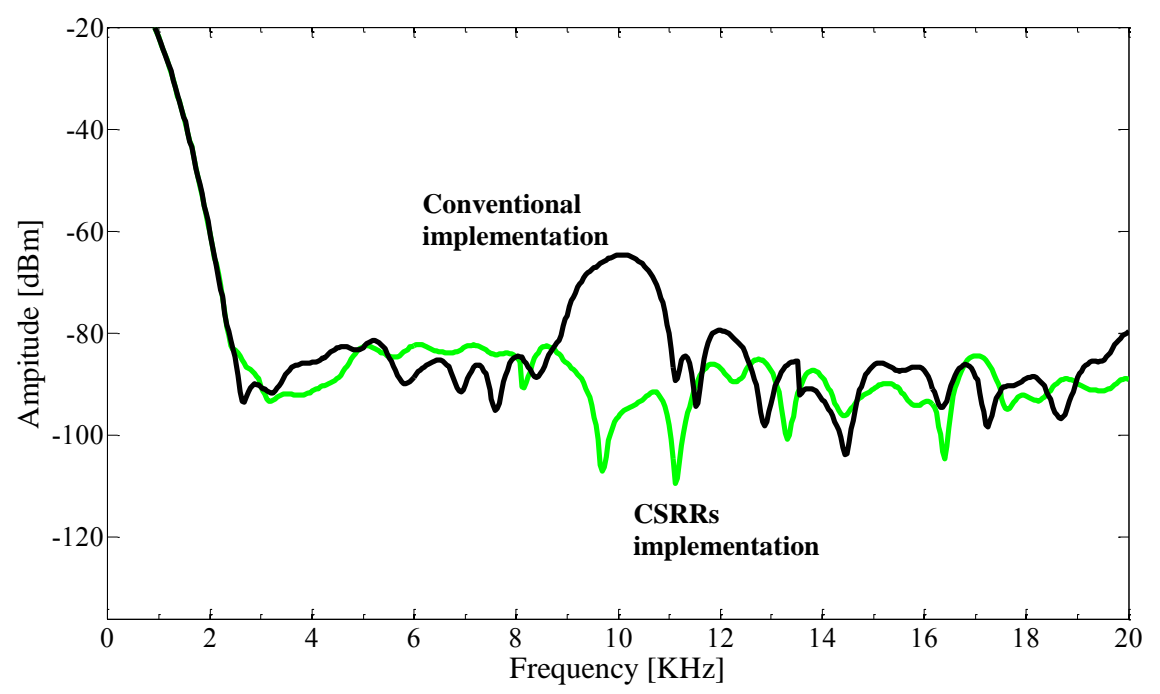


Fig. 13

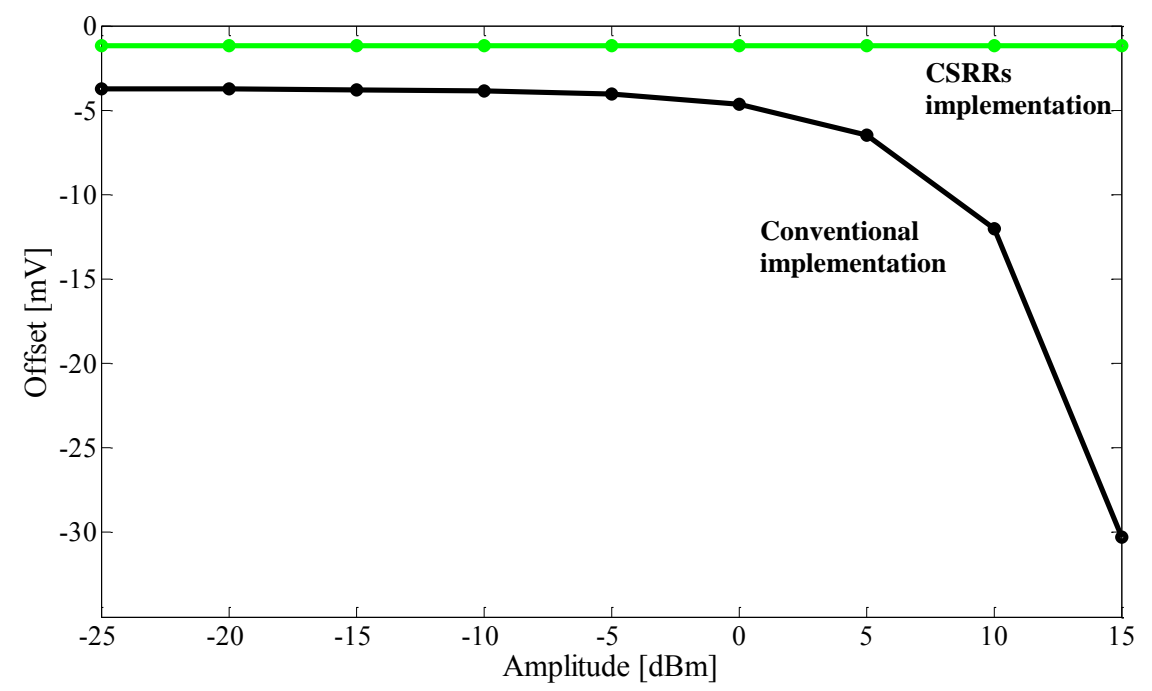


Fig. 14

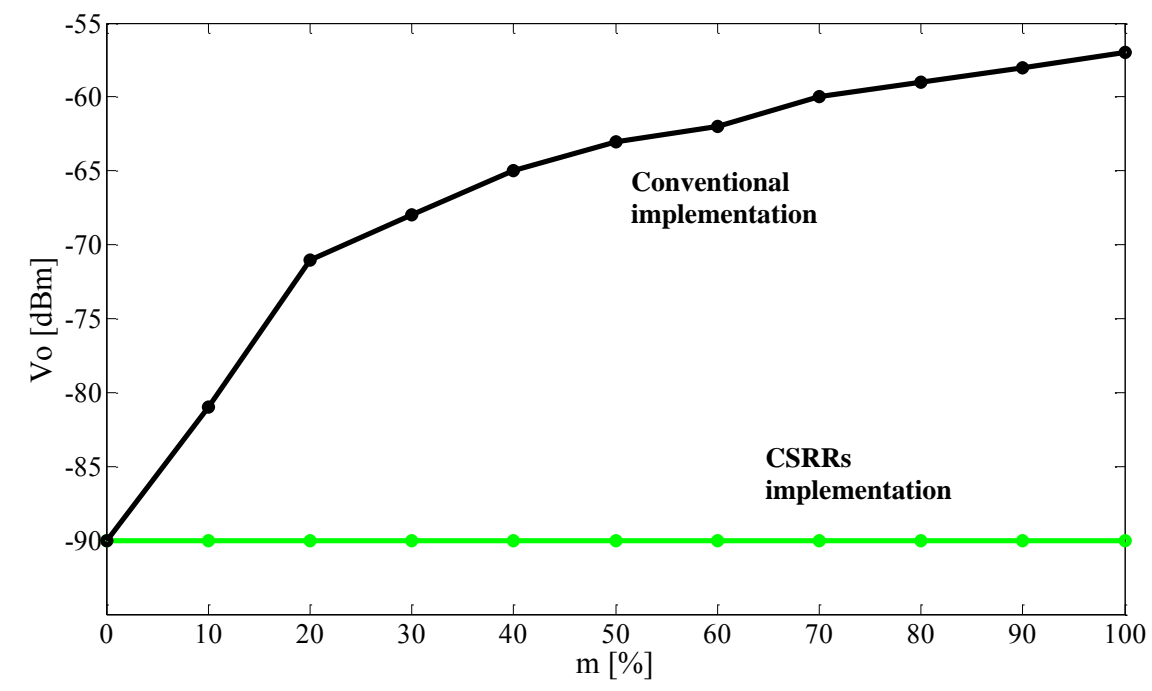


Fig. 15

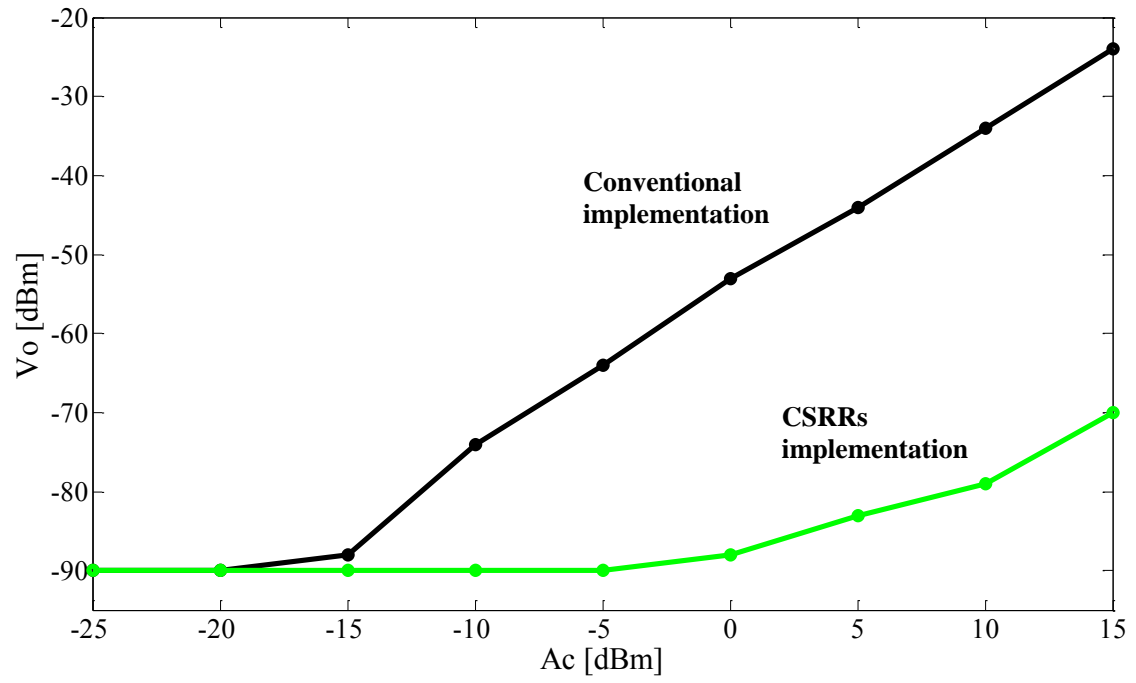

